\title{
Sums of distances to the nearest integer and the discrepancy of digital nets
}

\author{
by
}

\section{G. Larcher and F. Pillichshammer (Linz)}

1. Introduction. The concept of digital nets provides at the moment the most efficient method to generate point sets with small star-discrepancy $D_{N}^{*}$. For a set of points $\mathbf{x}_{0}, \ldots, \mathbf{x}_{N-1}$ in $[0,1)^{d}$ the star-discrepancy of the point set is defined by

$$
D_{N}^{*}=\sup _{B}\left|\frac{A_{N}(B)}{N}-\lambda(B)\right|,
$$

where the supremum is taken over all subintervals $B$ of $[0,1)^{d}$ of the form $B=\prod_{i=1}^{d}\left[0, b_{i}\right), 0<b_{i} \leq 1, A_{N}(B)$ denotes the number of $i$ with $\mathbf{x}_{i} \in B$ and $\lambda$ is the Lebesgue measure.

It is known that for any set of $N$ points in $[0,1)^{2}$ one has

$$
\frac{N D_{N}^{*}}{\log N} \geq 0.06
$$

(see for example [1]).

A digital $(0, s, 2)$-net in base 2 is a point set of $N=2^{s}$ points $\mathbf{x}_{0}, \ldots, \mathbf{x}_{N-1}$ in $[0,1)^{2}$ which is generated as follows. Choose two $s \times s$-matrices $C_{1}, C_{2}$ over $\mathbb{Z}_{2}$ with the following property: For every integer $k, 0 \leq k \leq s$, the system of the first $k$ rows of $C_{1}$ together with the first $s-k$ rows of $C_{2}$ is linearly independent over $\mathbb{Z}_{2}$. Then to construct $\mathbf{x}_{n}:=\left(x_{n}^{(1)}, x_{n}^{(2)}\right)$ for $0 \leq n \leq 2^{s}-1$, represent $n$ in base 2 :

$$
n=n_{s-1} 2^{s-1}+\ldots+n_{1} 2+n_{0},
$$

multiply $C_{i}$ with the vector of digits:

$$
C_{i}\left(n_{0}, \ldots, n_{s-1}\right)^{\mathrm{T}}=:\left(y_{1}^{(i)}, \ldots, y_{s}^{(i)}\right)^{\mathrm{T}} \in \mathbb{Z}_{2}^{s}
$$

2000 Mathematics Subject Classification: 11K06, 11K38, 42C10.

Key words and phrases: digital nets, discrepancy, Walsh series analysis, distance to the nearest integer.

Research of F. Pillichshammer supported by the Austrian Research Foundation (FWF), Project S 8305. 
and set

$$
x_{n}^{(i)}:=\sum_{j=1}^{s} \frac{y_{j}^{(i)}}{2^{j}} .
$$

It was shown by Niederreiter [8] that for the star-discrepancy of any digital $(0, s, 2)$-net in base 2 we have

$$
N D_{N}^{*} \leq \frac{1}{2} s+\frac{3}{2}
$$

hence

$$
\limsup _{N \rightarrow \infty} \max \frac{N D_{N}^{*}}{\log N} \leq \frac{1}{2 \log 2}=0.7213 \ldots
$$

where the maximum is taken over all digital $(0, s, 2)$-nets in base 2 with $N=2^{s}$ elements.

The simplest digital $(0, s, 2)$-net in base 2 is provided by choosing

$$
C_{1}=\left(\begin{array}{ccccc}
1 & 0 & \ldots & 0 & 0 \\
0 & 1 & \ldots & 0 & 0 \\
\ldots & \ldots & \ldots & \ldots & \ldots \\
0 & 0 & \ldots & 1 & 0 \\
0 & 0 & \ldots & 0 & 1
\end{array}\right) \quad \text { and } \quad C_{2}=\left(\begin{array}{ccccc}
0 & 0 & \ldots & 0 & 1 \\
0 & 0 & \ldots & 1 & 0 \\
\ldots & \ldots & \ldots & \ldots & \ldots \\
0 & 1 & \ldots & 0 & 0 \\
1 & 0 & \ldots & 0 & 0
\end{array}\right)
$$

This gives the well-known Hammersley point set in base 2 .

The star-discrepancy of this very special digital $(0, s, 2)$-net was studied by Halton and Zaremba [4], de Clerck [2] and Entacher [3]. The first two papers are very technical and very hard to read. Indeed in [4] an essential part of the proof (determining the extremal intervals) is not carried out in detail. [3] uses a new approach but also essentially relies on results from [4].

In this paper we study much more generally the star-discrepancy of digital $(0, s, 2)$-nets in base 2 .

In Section 2 (see Theorem 1) we give a compact explicit formula for the discrepancy function of digital $(0, s, 2)$-nets in base 2 . Our approach is via Walsh series analysis.

It turns out that this explicit formula is based on sums of distances to the nearest integer $(\|x\|:=\min (x-[x], 1-(x-[x])))$ of the form

$$
\sum_{u=0}^{s-1}\left\|2^{u} \beta\right\| \varepsilon_{u}
$$

with a real $\beta$ and certain integer sequences $\varepsilon_{u} \in\{-1,0,1\}$.

In Section 3 we study such sums on their own and we give a certain "spectrum" result for $\sum_{u=0}^{s-1}\left\|2^{u} \beta\right\|$ (see Theorems 2 and 3), part of which will be needed in Section 4.

In Section 4 we use the above results to study the Hammersley point set once more, to give a simple and now self-contained proof for the exact 
value of the "discrete discrepancy" and of the star-discrepancy of this point set (Theorem 4). Further we show that it is the "worst distributed" digital $(0, s, 2)$-net in base 2 with respect to star-discrepancy and we will get that for every digital $(0, s, 2)$-net in base 2 we have the (essentially) best possible bound

$$
N D_{N}^{*} \leq \frac{1}{3} s+\frac{19}{9}
$$

and that

$$
\lim _{N \rightarrow \infty} \max \frac{N D_{N}^{*}}{\log N}=\frac{1}{3 \log 2}=0.4808 \ldots
$$

(the maximum is taken over all digital $(0, s, 2)$-nets in base 2 with $N=2^{s}$ elements) with equality for the Hammersley point sets, thereby improving the bounds (1) and (2) of Niederreiter (Theorem 5).

Numerical investigations suggest that the minimal value for

$$
\limsup _{N \rightarrow \infty} \frac{N D_{N}^{*}}{\log N}
$$

over all digital $(0, s, 2)$-nets in base 2 is attained for the net generated by the matrices

$$
C_{1}=\left(\begin{array}{ccccc}
1 & 0 & \ldots & 0 & 0 \\
0 & 1 & \ldots & 0 & 0 \\
\ldots & \ldots & \ldots & \ldots & \ldots \\
0 & 0 & \ldots & 1 & 0 \\
0 & 0 & \ldots & 0 & 1
\end{array}\right) \quad \text { and } \quad C_{2}=\left(\begin{array}{ccccc}
1 & 1 & \ldots & 1 & 1 \\
1 & 1 & \ldots & 1 & 0 \\
\ldots \ldots & \ldots & \ldots & \ldots \\
1 & 1 & \ldots & 0 & 0 \\
1 & 0 & \ldots & 0 & 0
\end{array}\right)
$$

In Section 5 we give bounds for the star-discrepancy of this net and we show (Theorem 6) that for these nets

$$
\frac{N D_{N}^{*}}{\log N} \geq \frac{1}{5 \log 2}=0.2885 \ldots
$$

holds for all $N$ and that

$$
\limsup _{N \rightarrow \infty} \frac{N D_{N}^{*}}{\log N} \leq 0.32654 \ldots
$$

thereby answering a question of Entacher in [3, Section 4].

2. The discrepancy function of digital $(0, s, 2)$-nets. For $0 \leq \alpha, \beta$ $\leq 1$ we consider the discrepancy function

$$
\Delta(\alpha, \beta):=A_{N}([0, \alpha) \times[0, \beta))-N \alpha \beta
$$

for digital $(0, s, 2)$-nets $\mathbf{x}_{0}, \ldots, \mathbf{x}_{2^{s}-1}$ in base 2 (i.e. $\left.N=2^{s}\right)$.

Since the generating matrices $C_{1}, C_{2}$ of a $(0, s, 2)$-net must be regular, and since multiplying $C_{1}, C_{2}$ by a regular matrix $A$ does not change the point set (only its order) we may assume in all the following that 


$$
C_{1}=\left(\begin{array}{ccccc}
1 & 0 & \ldots & 0 & 0 \\
0 & 1 & \ldots & 0 & 0 \\
\ldots & \ldots & \ldots & \ldots & \ldots \\
0 & 0 & \ldots & 1 & 0 \\
0 & 0 & \ldots & 0 & 1
\end{array}\right) \quad \text { and } \quad C_{2}=\left(\begin{array}{cccc}
c_{1}^{1} & c_{1}^{2} & \ldots & c_{1}^{s} \\
c_{2}^{1} & c_{2}^{2} & \ldots & c_{2}^{s} \\
\ldots \ldots & \ldots \ldots & \ldots \\
c_{s}^{1} & c_{s}^{2} & \ldots & c_{s}^{s}
\end{array}\right)=:\left(\begin{array}{c}
\vec{c}_{1} \\
\vec{c}_{2} \\
\ldots \\
\vec{c}_{s}
\end{array}\right)
$$

We assume first that $\alpha$ and $\beta$ are "s-bit", i.e.

$$
\alpha=\frac{a_{1}}{2}+\ldots+\frac{a_{s}}{2^{s}}, \quad \beta=\frac{b_{1}}{2}+\ldots+\frac{b_{s}}{2^{s}} .
$$

For any $s$-bit number $\delta=d_{1} / 2+\ldots+d_{s} / 2^{s}$ we write

$$
\vec{\delta}:=\left(\begin{array}{c}
d_{1} \\
\vdots \\
d_{s}
\end{array}\right)
$$

and for a non-negative integer $k=k_{s-1} 2^{s-1}+\ldots+k_{1} 2+k_{0}$ we write

$$
\vec{k}:=\left(\begin{array}{c}
k_{0} \\
\vdots \\
k_{s-1}
\end{array}\right) \text {. }
$$

We need some further notation:

$$
\begin{gathered}
\vec{\gamma}:=\left(\begin{array}{c}
\gamma_{1} \\
\vdots \\
\gamma_{s}
\end{array}\right):=C_{2} \vec{\alpha}+\vec{\beta}, \quad \vec{\gamma}(u):=\left(\begin{array}{c}
\gamma_{1} \\
\vdots \\
\gamma_{u}
\end{array}\right), \\
C_{2}^{\prime}(u):=\left(\begin{array}{ccc}
c_{1}^{s-u+1} & \ldots & c_{u}^{s-u+1} \\
\ldots \ldots \ldots \ldots & -1 \\
c_{1}^{s} & \ldots & c_{u}^{s}
\end{array}\right)^{-1} .
\end{gathered}
$$

$\left(C_{2}^{\prime}(u)\right.$ exists since by the $(0, s, 2)$-net property the first $s-u$ rows of $C_{1}$ together with the first $u$ rows of $C_{2}$ must form a linearly independent system, hence the matrix

$$
C_{2}(u):=\left(\begin{array}{ccc}
c_{1}^{s-u+1} & \ldots & c_{1}^{s} \\
\ldots \ldots \ldots & \ldots & \ldots \\
c_{u}^{s-u+1} & \ldots & c_{u}^{s}
\end{array}\right)
$$

must be regular.) Note that $\gamma_{u}=\left(\vec{c}_{u} \mid \vec{\alpha}\right)+b_{u}$.

Further, for $0 \leq u \leq s-1$ let

$$
m(u):=\left\{\begin{array}{l}
0 \quad \text { if } u=0, \\
0 \quad \text { if }\left(\vec{\gamma}(u) \mid C_{2}^{\prime} \vec{e}_{1}\right)=1, \\
\max \left\{1 \leq j \leq u:\left(\vec{\gamma}(u) \mid C_{2}^{\prime} \vec{e}_{i}\right)=0 ; i=1, \ldots, j\right\} \quad \text { otherwise }
\end{array}\right.
$$

(here $(\cdot \mid \cdot)$ denotes the usual inner product in $\mathbb{Z}_{2}^{u}, \vec{e}_{i}$ is the $i$ th unit vector in $\mathbb{Z}_{2}^{u}$, and $\left.C_{2}^{\prime}:=C_{2}^{\prime}(u)\right)$.

Let $j(u):=u-m(u)$. Then we have 
Theorem 1. For all $\alpha, \beta$ s-bit, for the discrepancy function $\Delta(\alpha, \beta)$ of the digital $(0, s, 2)$-net in base 2 generated by

$$
C_{1}=\left(\begin{array}{ccccc}
1 & 0 & \ldots & 0 & 0 \\
0 & 1 & \ldots & 0 & 0 \\
\ldots & \ldots & \ldots & \ldots & \ldots \\
0 & 0 & \ldots & 1 & 0 \\
0 & 0 & \ldots & 0 & 1
\end{array}\right)
$$

and $C_{2}$ we have

$$
\begin{aligned}
\Delta(\alpha, \beta)= & \sum_{u=0}^{s-1}\left\|2^{u} \beta\right\|(-1)^{\left(\vec{c}_{u+1} \mid \vec{\alpha}\right)}(-1)^{\left(\vec{\gamma}(u) \mid C_{2}^{\prime}(u)\left(c_{u+1}^{s-u+1}, \ldots, c_{u+1}^{s}\right)^{\mathrm{T}}\right)} \\
& \times \frac{(-1)^{a_{s-u}}-(-1)^{a_{s+1-j(u)}}}{2}
\end{aligned}
$$

(here for $u=0$ we set $\left(\vec{\gamma}(u) \mid C_{2}^{\prime}(u)\left(c_{u+1}^{s-u+1}, \ldots, c_{u+1}^{s}\right)^{\mathrm{T}}\right)=0$ and $\left.a_{s+1}:=0\right)$.

Before we prove this result we give some remarks and examples.

REMARK 1. Note that $\Delta(\alpha, \beta)$ hence is of the form $\sum_{u=0}^{s-1}\left\|2^{u} \beta\right\| \varepsilon_{u}$ with some $\varepsilon_{u} \in\{-1,0,1\}$.

REMARK 2 . Let $0 \leq \alpha, \beta \leq 1$ now be arbitrary (not necessarily $s$-bit). Since all the points of the digital net have coordinates $x_{n}^{(i)}$ of the form $a / 2^{s}$ for some $a \in\left\{0,1, \ldots, 2^{s}-1\right\}$, we then have

$$
\Delta(\alpha, \beta)=\Delta(\alpha(s), \beta(s))+2^{s}(\alpha(s) \beta(s)-\alpha \beta)
$$

where $\alpha(s)$ (resp. $\beta(s)$ ) is the smallest $s$-bit number larger than or equal to $\alpha$ (resp. $\beta$ ).

EXAMPLE 1. Let $C_{2}$ be of triangular form

$$
C_{2}=\left(\begin{array}{ccccc}
c_{1}^{1} & c_{1}^{2} & \ldots & c_{1}^{s-1} & 1 \\
c_{2}^{1} & c_{2}^{2} & \ldots & 1 & 0 \\
\ldots \ldots & \ldots & \ldots & \ldots & \ldots \\
c_{s-1}^{1} & 1 & \ldots & 0 & 0 \\
1 & 0 & \ldots & 0 & 0
\end{array}\right)
$$

Then

$$
C_{2}^{\prime}(u)=\left(\begin{array}{ccccc}
0 & 0 & \ldots & 0 & 1 \\
0 & 0 & \ldots & 1 & d_{2}^{u} \\
\ldots & \ldots & \ldots & \ldots & \ldots \\
1 & d_{u}^{2} & \ldots & d_{u}^{u-1} & d_{u}^{u}
\end{array}\right)
$$

with certain $d_{i}^{j} \in \mathbb{Z}_{2}$. Hence

$$
C_{2}^{\prime}(u) \vec{e}_{i}=\left(0, \ldots, 0,1, d_{u+2-i}^{i}, \ldots, d_{u}^{i}\right)^{\mathrm{T}},
$$


and

$$
\left(\vec{\gamma}(u) \mid C_{2}^{\prime}(u) \vec{e}_{i}\right)=\gamma_{u+1-i}+\gamma_{u+2-i} d_{u+2-i}^{i}+\ldots+\gamma_{u} d_{u}^{i} .
$$

Therefore

$$
\begin{aligned}
& \max \left\{1 \leq j \leq u:\left(\vec{\gamma}(u) \mid C_{2}^{\prime}(u) \vec{e}_{i}\right)=0 ; i=1, \ldots, j\right\} \\
& \quad=\max \left\{1 \leq j \leq u: \gamma_{u+1-i}=0 ; i=1, \ldots, j\right\},
\end{aligned}
$$

hence $\gamma_{u}=\ldots=\gamma_{u+1-m(u)}=0, \gamma_{u-m(u)}=1$, so that

$$
j(u)=u-m(u)=\max \left\{j \leq u: \gamma_{j}=1\right\}=\max \left\{j \leq u:\left(\vec{c}_{j} \mid \vec{\alpha}\right) \neq b_{j}\right\} .
$$

Respectively

$$
j(u)= \begin{cases}0 & \text { if } u=0 \\ 0 & \text { if }\left(\vec{c}_{j} \mid \vec{\alpha}\right)=b_{j} \text { for } j=1, \ldots, u .\end{cases}
$$

Further $\left(c_{u+1}^{s-u+1}, \ldots, c_{u+1}^{s}\right)=(0, \ldots, 0)$, and so for $\alpha, \beta s$-bit we have

$$
\Delta(\alpha, \beta)=\sum_{u=0}^{s-1}\left\|2^{u} \beta\right\|(-1)^{\left(\vec{c}_{u+1} \mid \vec{\alpha}\right)} \frac{(-1)^{a_{s-u}}-(-1)^{a_{s+1-j(u)}}}{2} .
$$

EXAmPle 2. For the discrepancy function of the Hammersley point set, i.e. for the $(0, s, 2)$-net generated by

$$
C_{1}=\left(\begin{array}{ccccc}
1 & 0 & \ldots & 0 & 0 \\
0 & 1 & \ldots & 0 & 0 \\
\ldots & \ldots & \ldots & \ldots & \ldots \\
0 & 0 & \ldots & 1 & 0 \\
0 & 0 & \ldots & 0 & 1
\end{array}\right) \quad \text { and } \quad C_{2}=\left(\begin{array}{ccccc}
0 & 0 & \ldots & 0 & 1 \\
0 & 0 & \ldots & 1 & 0 \\
\ldots & \ldots & \ldots & \ldots & \ldots \\
0 & 1 & \ldots & 0 & 0 \\
1 & 0 & \ldots & 0 & 0
\end{array}\right)
$$

because of $\left(\vec{c}_{j} \mid \vec{\alpha}\right)=a_{s+1-j}$ we obtain (for $\alpha, \beta s$-bit)

$$
\begin{aligned}
\Delta(\alpha, \beta) & =\sum_{u=0}^{s-1}\left\|2^{u} \beta\right\| \frac{1-(-1)^{a_{s-u}+a_{s+1-j(u)}}}{2} \\
& =\sum_{u=0}^{s-1}\left\|2^{u} \beta\right\|\left(a_{s-u} \oplus a_{s+1-j(u)}\right)
\end{aligned}
$$

(where $\oplus$ denotes addition modulo 2 ).

EXAMPLE 3. For the discrepancy function of the $(0, s, 2)$-net generated by

$$
C_{1}=\left(\begin{array}{ccccc}
1 & 0 & \ldots & 0 & 0 \\
0 & 1 & \ldots & 0 & 0 \\
\ldots & \ldots & \ldots & \ldots & \ldots \\
0 & 0 & \ldots & 1 & 0 \\
0 & 0 & \ldots & 0 & 1
\end{array}\right) \quad \text { and } \quad C_{2}=\left(\begin{array}{ccccc}
1 & 1 & \ldots & 1 & 1 \\
1 & 1 & \ldots & 1 & 0 \\
\ldots & \ldots & \ldots & \ldots & \ldots \\
1 & 1 & \ldots & 0 & 0 \\
1 & 0 & \ldots & 0 & 0
\end{array}\right)
$$


because of $\left(\vec{c}_{j} \mid \vec{\alpha}\right)=a_{1} \oplus \ldots \oplus a_{s+1-j}$ we obtain (for $\alpha, \beta s$-bit)

$$
\Delta(\alpha, \beta)=\sum_{u=0}^{s-1}\left\|2^{u} \beta\right\|(-1)^{a_{1}+\ldots+a_{s-u}} \frac{(-1)^{a_{s-u}}-(-1)^{a_{s+1-j(u)}}}{2} .
$$

For the proof of the Theorem 1 we need two auxiliary results.

LemMa 1. Let $z$ be of the form $z=p / 2^{s}, p \in\left\{0, \ldots, 2^{s}-1\right\}$. Then for the characteristic function $\chi_{[0, z)}$ of the interval $[0, z)$ we have

$$
\chi_{[0, z)}(x)=\sum_{k=0}^{2^{s}-1} c_{k}(z) \operatorname{wal}_{k}(x)
$$

where wal $_{k}$ denotes the kth Walsh function in base 2 (see Remark 3 ),

$$
c_{k}(z)= \begin{cases}z & \text { if } k=0 \\ \operatorname{wal}_{k}(z) \frac{1}{2^{v(k)}} \psi\left(2^{v(k)} z\right) & \text { if } k \neq 0\end{cases}
$$

$\psi(x)$ is periodic with period 1 and

$$
\psi(x)= \begin{cases}x & \text { if } 0 \leq x<1 / 2 \\ x-1 & \text { if } 1 / 2 \leq x<1\end{cases}
$$

and $v(k)=r$ if $2^{r} \leq k<2^{r+1}$.

REMARK 3. Recall that Walsh functions in base 2 can be defined as follows: For a non-negative integer $k$ with base 2 representation $k=k_{m} 2^{m}+$ $\ldots+k_{1} 2+k_{0}$ and a real $x$ with (canonical) base 2 representation $x=$ $x_{1} / 2+x_{2} / 2^{2}+\ldots$ we have

$$
\operatorname{wal}_{k}(x)=(-1)^{x_{1} k_{0}+x_{2} k_{1}+\ldots+x_{m+1} k_{m}}=(-1)^{(\vec{k} \mid \vec{x})} .
$$

Proof of Lemma 1. This is a simple calculation, a proof can be found for example in [6, Lemma 2].

Lemma 2. Let $\psi$ be as in Lemma 1. Then

$$
\psi\left(2^{l+1} \beta\right)-\sum_{i=0}^{l} \psi\left(2^{i} \beta\right)=\{\beta\}-b_{l+2} .
$$

(Here $\{\beta\}=\beta-[\beta]$.

Proof. Let $\{\beta\}=\sum_{j=1}^{\infty} b_{j} 2^{-j}$. Then

$$
\psi\left(2^{i} \beta\right)=\sum_{j=i+1}^{\infty} b_{j} 2^{i-j}-b_{i+1}
$$


and therefore

$$
\begin{aligned}
\sum_{i=0}^{l} \psi\left(2^{i} \beta\right) & =\sum_{i=0}^{l}\left(\left(\sum_{j=i+1}^{\infty} b_{j} 2^{i-j}\right)-b_{i+1}\right) \\
& =\sum_{j=1}^{l+1} b_{j} 2^{-j} \sum_{i=0}^{j-1} 2^{i}+\sum_{j=l+2}^{\infty} b_{j} 2^{-j} \sum_{i=0}^{l} 2^{i}-\sum_{i=0}^{l} b_{i+1} \\
& =\sum_{j=l+2}^{\infty} b_{j} 2^{(l+1)-j}-\sum_{j=1}^{\infty} b_{j} 2^{-j}=\psi\left(2^{l+1} \beta\right)-\{\beta\}+b_{l+2} .
\end{aligned}
$$

Proof of Theorem 1. Let $I:=[0, \alpha) \times[0, \beta)$. Then for $\mathbf{y}=\left(y^{(1)}, y^{(2)}\right) \in$ $[0,1)^{2}$ by Lemma 1 we have

$$
\begin{aligned}
\chi_{I}(\mathbf{y})-\lambda(I)= & \chi_{[0, \alpha)}\left(y^{(1)}\right) \chi_{[0, \beta)}\left(y^{(2)}\right)-\alpha \beta \\
= & \sum_{\substack{k, l=0 \\
(k, l) \neq(0,0)}}^{2^{s}-1} c_{k}(\alpha) c_{l}(\beta) \operatorname{wal}_{k}\left(y^{(1)}\right) \operatorname{wal}_{l}\left(y^{(2)}\right) \\
= & \alpha \sum_{l=1}^{2^{s}-1} \operatorname{wal}_{l}(\beta) \frac{1}{2^{v(l)}} \psi\left(2^{v(l)} \beta\right) \operatorname{wal}_{l}\left(y^{(2)}\right) \\
& +\beta \sum_{k=1}^{2^{s}-1} \operatorname{wal}_{k}(\alpha) \frac{1}{2^{v(k)}} \psi\left(2^{v(k)} \alpha\right) \operatorname{wal}_{k}\left(y^{(1)}\right) \\
& +\sum_{k, l=1}^{2^{s}-1} \operatorname{wal}_{k}(\alpha) \operatorname{wal}_{l}(\beta) \frac{1}{2^{v(k)+v(l)}} \psi\left(2^{v(k)} \alpha\right) \psi\left(2^{v(l)} \beta\right) \\
& \times \operatorname{wal}_{k}\left(y^{(1)}\right) \operatorname{wal}_{l}\left(y^{(2)}\right) .
\end{aligned}
$$

Hence

$$
\begin{aligned}
\Delta(\alpha, \beta)= & \alpha \sum_{l=1}^{2^{s}-1} \operatorname{wal}_{l}(\beta) \frac{1}{2^{v(l)}} \psi\left(2^{v(l)} \beta\right) \sum_{i=0}^{2^{s}-1} \operatorname{wal}_{l}\left(y_{i}\right) \\
& +\beta \sum_{k=1}^{2^{s}-1} \operatorname{wal}_{k}(\alpha) \frac{1}{2^{v(k)}} \psi\left(2^{v(k)} \alpha\right) \sum_{i=0}^{2^{s}-1} \operatorname{wal}_{k}\left(x_{i}\right) \\
& +\sum_{k, l=1}^{2^{s}-1} \operatorname{wal}_{k}(\alpha) \operatorname{wal}_{l}(\beta) \frac{\psi\left(2^{v(k)} \alpha\right) \psi\left(2^{v(l)} \beta\right)}{2^{v(k)+v(l)}} \sum_{i=0}^{2^{s}-1} \operatorname{wal}_{k}\left(x_{i}\right) \operatorname{wal}_{l}\left(y_{i}\right) .
\end{aligned}
$$

(Here the net consists of the points $\mathbf{x}_{i}, i=0, \ldots, 2^{s}-1$, with $\mathbf{x}_{i}:=\left(x_{i}, y_{i}\right)$.)

Since $\mathbf{x}_{i}, i=0, \ldots, 2^{s}-1$, is a digital $(0, s, 2)$-net, for all $0<k, l<2^{s}$ we have 


$$
\sum_{i=0}^{2^{s}-1} \mathrm{wal}_{k}\left(x_{i}\right)=\sum_{i=0}^{2^{s}-1} \mathrm{wal}_{l}\left(y_{i}\right)=0
$$

(see for example [5, Lemma 2]).

We now consider $\sum_{i=0}^{2^{s}-1} \operatorname{wal}_{k}\left(x_{i}\right) \operatorname{wal}_{l}\left(y_{i}\right)$ with $x_{i}:=x_{i}^{(1)} / 2+\ldots+x_{i}^{(s)} / 2^{s}$ and $y_{i}:=y_{i}^{(1)} / 2+\ldots+y_{i}^{(s)} / 2^{s}$. We identify $\left(x_{i}, y_{i}\right)$ with

$$
\left(x_{i}^{(1)}, \ldots, x_{i}^{(s)}, y_{i}^{(1)}, \ldots, y_{i}^{(s)}\right)^{\mathrm{T}} \in\left(\mathbb{Z}_{2}\right)^{2 s}
$$

and we define

$$
\left(x_{i}, y_{i}\right) \oplus\left(x_{i}^{\prime}, y_{i}^{\prime}\right):=\left(x_{i}^{(1)}+x_{i}^{\prime(1)}, \ldots, y_{i}^{(s)}+y_{i}^{\prime(s)}\right) .
$$

Further $\operatorname{wal}_{k, l}\left(x_{i}, y_{i}\right):=\operatorname{wal}_{k}\left(x_{i}\right) \operatorname{wal}_{l}\left(y_{i}\right)$, hence

$$
\operatorname{wal}_{k, l}\left(\left(x_{i}, y_{i}\right) \oplus\left(x_{i}^{\prime}, y_{i}^{\prime}\right)\right)=\operatorname{wal}_{k, l}\left(x_{i}, y_{i}\right) \operatorname{wal}_{k, l}\left(x_{i}^{\prime}, y_{i}^{\prime}\right),
$$

i.e. wal $_{k, l}$ is a character on $\left(\left(\mathbb{Z}_{2}\right)^{2 s}, \oplus\right)$.

The digital net $\mathbf{x}_{0}, \ldots, \mathbf{x}_{2^{s}-1}$ is a subgroup of $\left(\left(\mathbb{Z}_{2}\right)^{2 s}, \oplus\right)$, hence

$$
\sum_{i=0}^{2^{s}-1} \operatorname{wal}_{k}\left(x_{i}\right) \text { wal }_{l}\left(y_{i}\right)= \begin{cases}2^{s} & \text { if } \text { wal }_{k, l}\left(x_{i}, y_{i}\right)=1 \text { for all } i=0, \ldots, 2^{s}-1, \\ 0 & \text { otherwise. }\end{cases}
$$

(For more details see [5] or [7].)

Now $\operatorname{wal}_{k, l}\left(x_{i}, y_{i}\right)=(-1)^{\left(\vec{k} \mid \vec{x}_{i}\right)+\left(\vec{l} \mid \vec{y}_{i}\right)}=1$ for all $i=0, \ldots, 2^{s}-1$ iff

$$
\left(\vec{k} \mid \vec{x}_{i}\right)=\left(\vec{l} \mid \vec{y}_{i}\right) \quad \text { for all } i=0, \ldots, 2^{s}-1,
$$

(by the definition of the net) this means

$$
(\vec{k} \mid \vec{i})=\left(\vec{l} \mid C_{2} \vec{i}\right) \quad \text { for all } i=0, \ldots, 2^{s}-1,
$$

and this is satisfied if and only if

$$
\vec{k}=C_{2}^{\mathrm{T}} \vec{l}=: \vec{k}(l) .
$$

Further

$$
\operatorname{wal}_{k(l)}(\alpha) \operatorname{wal}_{l}(\beta)=(-1)^{(\vec{k}(l) \mid \vec{\alpha})+(\vec{l} \mid \vec{\beta})}=(-1)^{\left(\vec{l} \mid C_{2} \vec{\alpha}+\vec{\beta}\right)}=\operatorname{wal}_{l}(\gamma)
$$

(see notations).

So

$$
\begin{aligned}
\Delta(\alpha, \beta)= & 2^{s} \sum_{u=0}^{s-1} \frac{\psi\left(2^{u} \beta\right)}{2^{u}} \sum_{l=2^{u}}^{2^{u+1}-1}(-1)^{l_{0} \gamma_{1}+\ldots+l_{u-1} \gamma_{u}+\gamma_{u+1}} \frac{\psi\left(2^{v(k(l))} \alpha\right)}{2^{v(k(l))}} \\
= & 2^{s} \sum_{u=0}^{s-1}\left\|2^{u} \beta\right\|(-1)^{\left(\vec{c}_{u+1} \mid \vec{\alpha}\right)} \\
& \times \frac{1}{2^{u}} \sum_{l=2^{u}}^{2^{u+1}-1}(-1)^{l_{0} \gamma_{1}+\ldots+l_{u-1} \gamma_{u}} \frac{\psi\left(2^{v(k(l))} \alpha\right)}{2^{v(k(l))}}
\end{aligned}
$$


(here $l:=l_{0}+l_{1} 2+\ldots+l_{u} 2^{u}$; note that $(-1)^{\gamma_{u+1}}=(-1)^{\left(\vec{c}_{u+1} \mid \vec{\alpha}\right)}(-1)^{b_{u+1}}$ and $\left.\psi\left(2^{u} \beta\right)(-1)^{b_{u+1}}=\left\|2^{u} \beta\right\|\right)$.

We now consider

$$
\begin{aligned}
\Sigma_{1} & :=\frac{1}{2^{u}} \sum_{l=2^{u}}^{2^{u+1}-1}(-1)^{l_{0} \gamma_{1}+\ldots+l_{u-1} \gamma_{u}} \frac{\psi\left(2^{v(k(l))} \alpha\right)}{2^{v(k(l))}} \\
& =\frac{1}{2^{u}} \sum_{w=0}^{s-1} \frac{\psi\left(2^{w} \alpha\right)}{2^{w}} \sum_{\substack{l=2^{u} \\
v(k(l))=w}}^{2^{u+1}-1}(-1)^{l_{0} \gamma_{1}+\ldots+l_{u-1} \gamma_{u}} .
\end{aligned}
$$

For $2^{u} \leq l<2^{u+1}$, the condition $v(k(l))=w$ means that there are $k_{0}, \ldots$, $k_{w-1} \in \mathbb{Z}_{2}$ such that

$$
C_{2}^{\mathrm{T}} \vec{l}=\left(k_{0}, \ldots, k_{w-1}, 1,0, \ldots, 0\right)^{\mathrm{T}},
$$

that is,

$$
\vec{c}_{1} l_{0}+\ldots+\vec{c}_{u} l_{u-1}+\vec{c}_{u+1}=k_{0} \vec{e}_{1}+\ldots+k_{w-1} \vec{e}_{w}+\vec{e}_{w+1}
$$

where $\vec{e}_{i}$ is the $i$ th unit vector in $\mathbb{Z}_{2}^{s}$.

Since $\vec{c}_{1}, \ldots, \vec{c}_{u+1}, \vec{e}_{1}, \ldots, \vec{e}_{w+1}$ by the $(0, s, 2)$-net property are linearly independent as long as $(u+1)+(w+1) \leq s$ we must have $u+w \geq s-1$. Hence

$$
\Sigma_{1}=\sum_{w=s-1-u}^{s-1} \frac{\psi\left(2^{w} \alpha\right)}{2^{u+w}} \sum_{\substack{l=2^{u} \\ v(k(l))=w}}^{2^{u+1}-1}(-1)^{l_{0} \gamma_{1}+\ldots+l_{u-1} \gamma_{u}} .
$$

In the following we are concerned with evaluating the last sum in the above expression which equals

$$
\Sigma_{2}:=\sum_{\substack{l=0 \\ v\left(k\left(l+2^{u}\right)\right)=w}}^{2^{u}-1} \operatorname{wal}_{l}(\gamma)=\sum_{\substack{C^{\prime} l=0 \\ v\left(k\left(C_{2}^{\prime} l+2^{u}\right)\right)=w}}^{2^{u}-1} \operatorname{wal}_{C_{2}^{\prime} l}(\gamma)
$$

(here $C_{2}^{\prime}$ stands for $C_{2}^{\prime}(u)$; see notation). Now $v\left(k\left(C_{2}^{\prime} l+2^{u}\right)\right)=w$ means

$$
C_{2}^{\mathrm{T}}\left(\begin{array}{c}
C_{2}^{\prime} \vec{l} \\
1 \\
0 \\
\vdots \\
0
\end{array}\right)=\left(\begin{array}{c}
k_{0} \\
\vdots \\
k_{w-1} \\
1 \\
0 \\
\vdots \\
0
\end{array}\right)
$$

for some $k_{i} \in \mathbb{Z}_{2}$. This is equivalent to 
(6)

$$
\left(\begin{array}{ccccc}
\multicolumn{5}{c}{D} \\
\\
1 & 0 & \ldots & 0 & 0 \\
0 & 1 & \ldots & 0 & 0 \\
\ldots \ldots \ldots \ldots & \ldots \\
0 & 0 & \ldots & 1 & 0 \\
0 & 0 & \ldots & 0 & 1
\end{array}\right) \vec{l}=\left(\begin{array}{c}
k_{0} \\
\vdots \\
k_{w-1} \\
1 \\
0 \\
\vdots \\
0
\end{array}\right)+\left(\begin{array}{c}
c_{u+1}^{1} \\
\vdots \\
c_{u+1}^{s}
\end{array}\right)
$$

with

$$
D=\left(\begin{array}{ccc}
c_{1}^{1} & \ldots & c_{u}^{1} \\
\ldots \ldots & \ldots & \ldots \\
c_{1}^{s-u} & \ldots & c_{u}^{s-u}
\end{array}\right) C_{2}^{\prime},
$$

i.e. an $(s-u) \times u$-matrix.

Let $s-u=w+1$. We first show that in this case equation (6) has a solution $\vec{l}$. This is equivalent to showing that system (5) has a solution, i.e., that there are $l_{0}, \ldots, l_{u-1}, k_{0}, \ldots, k_{w-1}$ in $\mathbb{Z}_{2}$ such that

$$
\vec{c}_{1} l_{0}+\ldots+\vec{c}_{u} l_{u-1}+\vec{c}_{u+1}+\vec{e}_{1} k_{0}+\ldots+\vec{e}_{w} k_{w-1}+\vec{e}_{w+1}=0 .
$$

Since $s=u+w+1$ the vectors $\vec{c}_{1}, \ldots, \vec{c}_{u+1}, \vec{e}_{1}, \ldots, \vec{e}_{w+1}$ are linearly dependent, and hence we can find $l_{0}, \ldots, l_{u-1}, l_{u}, k_{0}, \ldots, k_{w-1}, k_{w}$ in $\mathbb{Z}_{2}$ not all zero such that

$$
\vec{c}_{1} l_{0}+\ldots+\vec{c}_{u} l_{u-1}+\vec{c}_{u+1} l_{u}+\vec{e}_{1} k_{0}+\ldots+\vec{e}_{w} k_{w-1}+\vec{e}_{w+1} k_{w}=0 .
$$

Assume that $l_{u}=0$. Then $\vec{c}_{1}, \ldots, \vec{c}_{u}, \vec{e}_{1}, \ldots, \vec{e}_{w+1}$ are linearly dependent. But this contradicts the $(0, s, 2)$-net property since $\vec{c}_{1}, \ldots, \vec{c}_{u}$ are the first $u$ rows of the matrix $C_{2}$ and $\vec{e}_{1}, \ldots, \vec{e}_{w+1}$ are the first $w+1$ rows of the matrix $C_{1}$ and $u+w+1=s$. Hence $l_{u}=1$. In the same way one can show that $k_{w}=1$. This shows that system (5), and hence also (6), has a solution.

Now the unique solution $\vec{l}$ of $(6)$ is given by

$$
\vec{l}=\left(c_{u+1}^{s-u+1}, \ldots, c_{u+1}^{s}\right)^{\mathrm{T}} .
$$

If $s-u \leq w$, then the $2^{u+w-s}$ solutions therefore are given by

$$
\vec{l}=\left(l_{0}, \ldots, l_{u+w-(s+1)}, c_{u+1}^{w+1} \oplus 1, c_{u+1}^{w+2}, \ldots, c_{u+1}^{s}\right)^{\mathrm{T}}
$$

with $l_{0}, \ldots, l_{u+w-(s+1)}$ arbitrary in $\mathbb{Z}_{2}$.

Hence for $w \geq s-u$ we have

$$
\begin{aligned}
\Sigma_{2} & =\sum_{l_{0}, \ldots, l_{u+w-(s+1)} \in \mathbb{Z}_{2}}(-1)^{\left(\vec{\gamma}(u) \mid C_{2}^{\prime}\left(l_{0}, \ldots, l_{u+w-(s+1)}, c_{u+1}^{w+1} \oplus 1, c_{u+1}^{w+2}, \ldots, c_{u+1}^{s}\right)^{\mathrm{T}}\right)} \\
& =(-1)^{\left(C_{2}^{\prime \mathrm{T}} \vec{\gamma}(u) \mid\left(0, \ldots, 0, c_{u+1}^{w+1} \oplus 1, c_{u+1}^{w+2}, \ldots, c_{u+1}^{s}\right)^{\mathrm{T}}\right)} \sum_{l=0}^{2^{u+w-s}-1} \operatorname{wal}_{l}\left(C_{2}^{\prime \mathrm{T}} \gamma(u)\right) .
\end{aligned}
$$


The last sum is a sum over all characters of $\left(\left(\mathbb{Z}_{2}\right)^{u+w-s}, \oplus\right)$, and is therefore $2^{u+w-s}$ if $\left(C_{2}^{\prime \mathrm{T}} \vec{\gamma}(u) \mid \vec{e}_{i}\right)=0$ for all $i=1, \ldots, u+w-s\left(\vec{e}_{i}\right.$ is the $i$ th unit vector in $\left.\mathbb{Z}_{2}^{u}\right)$ and it is 0 otherwise.

Further, if $\left(C_{2}^{\prime \mathrm{T}} \vec{\gamma}(u) \mid \vec{e}_{i}\right)=0$ for all $i=1, \ldots, u+w-s$ (we will call this the condition $\left.*_{u}\right)$, then

$$
\begin{aligned}
\left(C_{2}^{\prime \mathrm{T}} \vec{\gamma}(u) \mid(0, \ldots,\right. & \left.\left.0, c_{u+1}^{w+1} \oplus 1, c_{u+1}^{w+2}, \ldots, c_{u+1}^{s}\right)^{\mathrm{T}}\right) \\
& =\left(\vec{\gamma}(u) \mid C_{2}^{\prime}\left(c_{u+1}^{s-u+1}, \ldots, c_{u+1}^{s}\right)^{\mathrm{T}}\right)+\left(\vec{\gamma}(u) \mid C_{2}^{\prime} \vec{e}_{u+w-s+1}\right),
\end{aligned}
$$

so that altogether we have

$$
\Sigma_{1}=\frac{1}{2^{s}}(-1)^{\left(\vec{\gamma}(u) \mid C_{2}^{\prime}\left(c_{u+1}^{s-u+1}, \ldots, c_{u+1}^{s}\right)^{\mathrm{T}}\right)} f(u),
$$

where

$$
\begin{aligned}
f(u):= & 2 \psi\left(2^{s-u-1} \alpha\right) \\
& + \begin{cases}\sum_{w=s-u}^{s-1} \psi\left(2^{w} \alpha\right)(-1)^{\left(\vec{\gamma}(u) \mid C_{2}^{\prime} \vec{e}_{u+w-s+1}\right)} & \text { if } *_{u} \text { holds } \\
0 & \text { otherwise }\end{cases}
\end{aligned}
$$

and therefore

$$
\Delta(\alpha, \beta)=\sum_{u=0}^{s-1}\left\|2^{u} \beta\right\|(-1)^{\left(\vec{c}_{u+1} \mid \vec{\alpha}\right)}(-1)^{\left(\vec{\gamma}(u) \mid C_{2}^{\prime}\left(c_{u+1}^{s-u+1}, \ldots, c_{u+1}^{s}\right)^{\mathrm{T}}\right)} f(u) .
$$

It remains to show that

$$
f(u)=\frac{(-1)^{a_{s-u}}-(-1)^{a_{s+1-j(u)}}}{2} .
$$

By the definition of $m(u)$ we have $\left(\vec{\gamma}(u) \mid C_{2}^{\prime} \vec{e}_{1}\right)=\ldots=\left(\vec{\gamma}(u) \mid C_{2}^{\prime} \vec{e}_{m(u)}\right)=0$ and $\left(\vec{\gamma}(u) \mid C_{2}^{\prime} \vec{e}_{m(u)+1}\right)=1$, hence $* u$ holds iff $u+w-s \leq m(u)$. So finally

$$
\begin{aligned}
f(u) & =2 \psi\left(2^{s-u-1} \alpha\right)+\sum_{w=s-u}^{s-u+m(u)} \psi\left(2^{w} \alpha\right)(-1)^{\left(\vec{\gamma}(u) \mid C_{2}^{\prime} \vec{e}_{u+w-s+1}\right)} \\
& =2 \psi\left(2^{s-u-1} \alpha\right)+\sum_{w=s-u}^{s-u+m(u)-1} \psi\left(2^{w} \alpha\right)-\psi\left(2^{s-u+m(u)} \alpha\right) \\
& =\psi\left(2^{s-u-1} \alpha\right)-\sum_{w=0}^{s-u-2} \psi\left(2^{w} \alpha\right)+\sum_{w=0}^{s-u+m(u)-1} \psi\left(2^{w} \alpha\right)-\psi\left(2^{s-u+m(u)} \alpha\right) \\
& =\alpha-a_{s-u}-\alpha+a_{s+1-(u-m(u))}=a_{s+1-(u-m(u))}-a_{s-u} \\
& =\frac{(-1)^{a_{s-u}}-(-1)^{a_{s+1-j(u)}}}{2}
\end{aligned}
$$

where we used Lemma 2 and $j(u)=u-m(u)$. The result follows. 


\section{A spectrum result for sums of distances to the nearest inte-} ger. Here we study sums of the form $\sum_{u=0}^{s-1}\left\|2^{u} \beta\right\|$ for $\beta \in \mathbb{R}$, especially for $s$-bit $\beta$, and we derive results which are of independent interest and/or will be used in Section 4.

The essential technical tool is provided by

Lemma 3. Assume that $\beta=0 . b_{1} b_{2} \ldots$ (this always means base 2 representation) has two equal consecutive digits $b_{i} b_{i+1}$ with $i \leq s-1$ and let $i$ be minimal with this property, i.e.

$$
\begin{array}{ll}
\beta=0.01 \ldots 0100 b_{i+2} \ldots & \text { or } \\
\beta=0.10 \ldots 0100 b_{i+2} \ldots & \text { or } \\
\beta=0.01 \ldots 1011 b_{i+2} \ldots & \text { or } \\
\beta=0.10 \ldots 1011 b_{i+2} \ldots &
\end{array}
$$

Replace $\beta$ by

$$
\begin{array}{ll}
\gamma=0.10 \ldots 1010 b_{i+2} \ldots & \text { resp. } \\
\gamma=0.01 \ldots 1010 b_{i+2} \ldots & \text { resp. } \\
\gamma=0.10 \ldots 0101 b_{i+2} \ldots & \text { resp. } \\
\gamma=0.01 \ldots 0101 b_{i+2} \ldots &
\end{array}
$$

Then

$$
\sum_{u=0}^{s-1}\left\|2^{u} \gamma\right\|=\sum_{u=0}^{s-1}\left\|2^{u} \beta\right\|+ \begin{cases}\frac{1}{3}\left(1-(-1)^{i} / 2^{i}\right)(1-\tau) & \text { in the first two cases } \\ \frac{1}{3}\left(1-(-1)^{i} / 2^{i}\right) \tau & \text { in the last two cases }\end{cases}
$$

where $\tau:=0 . b_{i+2} b_{i+3} \ldots$

REMARK 4 . In any case we have $\sum_{u=0}^{s-1}\left\|2^{u} \gamma\right\| \geq \sum_{u=0}^{s-1}\left\|2^{u} \beta\right\|$ with equality iff $\tau=1$ in the first two cases and iff $\tau=0$ in the last two cases.

Proof of Lemma 3. This is simple calculation. We just handle the first case here:

$$
\begin{aligned}
& \sum_{u=0}^{s-1}\left(\left\|2^{u} \gamma\right\|-\left\|2^{u} \beta\right\|\right) \\
& \quad=\|\gamma\|-\left\|2^{i} \beta\right\|+\left(\left(\frac{\tau}{2}-\frac{\tau}{4}\right)-\left(\frac{\tau}{4}-\frac{\tau}{8}\right) \pm \ldots+\left(\frac{\tau}{2^{i}}-\frac{\tau}{2^{i+1}}\right)\right) \\
& \quad=\left(\frac{1}{3}\left(1+\frac{1}{2^{i}}\right)-\frac{\tau}{2^{i+1}}\right)-\frac{\tau}{2}+\frac{1}{6}\left(1+\frac{1}{2^{i}}\right) \tau \\
& \quad=\frac{1}{3}\left(1+\frac{1}{2^{i}}\right)(1-\tau) .
\end{aligned}
$$

The other cases are calculated in the same way.

We immediately obtain a corollary which is useful in Section 4. 
Corollary 1. Assume that $\beta=0.1 b_{2} b_{3} \ldots$ has two equal consecutive digits $b_{i} b_{i+1}$ with $2 \leq i \leq s-1$ and let $i$ be the minimal index with this property, i.e.

$$
\begin{array}{ll}
\beta=0.101 \ldots 0100 b_{i+2} \ldots & \text { or } \\
\beta=0.110 \ldots 0100 b_{i+2} \ldots & \text { or } \\
\beta=0.101 \ldots 1011 b_{i+2} \ldots & \text { or } \\
\beta=0.110 \ldots 1011 b_{i+2} \ldots &
\end{array}
$$

Replace $\beta$ by

$$
\begin{array}{llr}
\gamma=0.110 \ldots 1010 b_{i+2} \ldots & \text { resp. } \\
\gamma=0.101 \ldots 1010 b_{i+2} \ldots & \text { resp. } \\
\gamma=0.110 \ldots 0101 b_{i+2} \ldots & \text { resp. } \\
\gamma=0.101 \ldots 0101 b_{i+2} \ldots &
\end{array}
$$

Then

$$
\begin{aligned}
\gamma & +\sum_{u=0}^{s-1}\left\|2^{u} \gamma\right\| \\
& =\beta+\sum_{u=0}^{s-1}\left\|2^{u} \beta\right\|+ \begin{cases}\frac{1}{3}\left(1-(-1)^{i-1} / 2^{i-1}\right)(1-\tau) & \text { in the first two cases, } \\
\frac{1}{3}\left(1-(-1)^{i-1} / 2^{i-1}\right) \tau & \text { in the last two cases, }\end{cases}
\end{aligned}
$$

where $\tau:=0 . b_{i+2} b_{i+3} \cdots$

Proof. This follows from $\beta+\|\beta\|=\gamma+\|\gamma\|=1$, by applying Lemma 3 to $\beta^{\prime}:=0 . b_{2} b_{3} \ldots$

We obtain

TheOREM 2. Consider $\beta \in \mathbb{R}$ with the canonical base 2 representation (i.e. with infinitely many digits equal to zero). Then there exists

$$
\max _{\beta} \sum_{u=0}^{s-1}\left\|2^{u} \beta\right\|=\frac{s}{3}+\frac{1}{9}-(-1)^{s} \frac{1}{9 \cdot 2^{s}}
$$

and it is attained if and only if $\beta$ is of the form $\beta_{0}$ with

$$
\beta_{0}=\frac{2}{3}\left(1-\left(-\frac{1}{2}\right)^{s+1}\right) \quad \text { or } \quad \beta_{0}=\frac{1}{3}\left(1-\left(-\frac{1}{2}\right)^{s}\right) \text {. }
$$

REMARK 5. Note that

$$
\begin{gathered}
\frac{2}{3}\left(1-\left(-\frac{1}{2}\right)^{s+1}\right)= \begin{cases}0.1010 \ldots 101 & \text { if } s \text { is odd } \\
0.1010 \ldots 011 & \text { if } s \text { is even, }\end{cases} \\
\frac{1}{3}\left(1-\left(-\frac{1}{2}\right)^{s}\right)= \begin{cases}0.0101 \ldots 011 & \text { if } s \text { is odd } \\
0.0101 \ldots 101 & \text { if } s \text { is even. }\end{cases}
\end{gathered}
$$

Proof of Theorem 2. For any $\gamma=0 . c_{1} c_{2} \ldots c_{s} c_{s+1} \ldots$ with fixed $c_{1}, \ldots, c_{s}$ the sum $\sum_{u=0}^{s-1}\left\|2^{u} \gamma\right\|$ obviously becomes maximal if $c_{s}=0$ and $c_{s+1}=$ 
$c_{s+2}=\ldots=1$, or if $c_{s}=1$ and $c_{s+1}=c_{s+2}=\ldots=0$. Hence by Lemma 3 the supremum

$$
\sup _{\beta} \sum_{u=0}^{s-1}\left\|2^{u} \beta\right\|
$$

can only be attained, respectively approached by

$$
\begin{aligned}
\beta_{1}= & 0.1010 \ldots 10111 \ldots \text { or } \\
& \left(b_{s} \text { is the last zero }\right) \\
\beta_{2}= & 0.0101 \ldots 01 \text { or } \\
\beta_{3}= & 0.1010 \ldots 11 \\
& \left(b_{s} \text { is the last one }\right)
\end{aligned}
$$

if $s$ is even, and by

$$
\begin{aligned}
& \beta_{4}=0.0101 \ldots 10111 \ldots \quad \text { or } \\
& \beta_{5}=0.1010 \ldots 01 \quad \text { or } \\
& \beta_{6}=0.0101 \ldots 11
\end{aligned}
$$

if $s$ is odd.

Now we check easily that

$$
\sum_{u=0}^{s-1}\left\|2^{u} \beta_{i}\right\|=\frac{s}{3}+\frac{1}{9}-(-1)^{s} \frac{1}{9 \cdot 2^{s}}
$$

for $i=1, \ldots, 6$ and the result follows.

The next theorem gives the result which we call the "spectrum" result (see Remark 6).

Theorem 3. (a) The maximum

$$
\max _{\beta s-b i t} \sum_{u=0}^{s-1}\left\|2^{u} \beta\right\|=\frac{s}{3}+\frac{1}{9}-(-1)^{s} \frac{1}{9 \cdot 2^{s}}
$$

is attained if and only if $\beta$ is one of the $\beta_{0}$ from Theorem 2 .

(b) We have

$$
\max _{\substack{\beta s-b i t \\ \beta \neq \beta_{0}}} \sum_{u=0}^{s-1}\left\|2^{u} \beta\right\|=\frac{s}{3}+\frac{1}{36}-(-1)^{s} \frac{7}{9 \cdot 2^{s}}
$$

and this second successive maximum is attained if and only if $\beta$ is of the form $\beta^{\prime}$ with

$$
\beta^{\prime}= \begin{cases}0.01101010 \ldots 101 & \text { or } \\ 0.010101 \ldots 01101 & \text { or } \\ 0.10010101 \ldots 011 & \end{cases}
$$


if $s$ is odd and

if $s$ is even.

$$
\beta^{\prime}= \begin{cases}0.100101010 \ldots 101 & \text { or } \\ 0.010101 \ldots 010011 & \text { or } \\ 0.101010 \ldots 101101 & \text { or } \\ 0.011010 \ldots 101011 & \end{cases}
$$

REMARK 6. Let

$$
\max _{\beta \text {-bit }} \sum_{u=0}^{s-1}\left\|2^{u} \beta\right\|=: \sum_{u=0}^{s-1}\left\|2^{u} \beta_{0}(s)\right\| .
$$

Then by Theorem 3 we have

$$
\lim _{s \rightarrow \infty}\left(\sum_{u=0}^{s-1}\left\|2^{u} \beta_{0}(s)\right\|-\max _{\substack{\beta s \text {-bit } \\ \beta \neq \beta_{0}(s)}} \sum_{u=0}^{s-1}\left\|2^{u} \beta\right\|\right)=\frac{1}{12} .
$$

So one may ask the further usual "spectrum questions".

Proof of Theorem 3. (a) follows from Theorem 2.

Concerning part (b) it follows from Lemma 3 that it must be possible to reach one of the $\beta_{0}$ by applying a single transformation of Lemma 3 to $\beta^{\prime}$.

For $s$ odd this means ( $s$ even is handled quite analogously) that

$$
\beta^{\prime} \rightarrow 0.1010 \ldots 101
$$

by the first or third transformation, i.e.

$$
\begin{aligned}
& \beta^{\prime}=0.0101 \ldots 01001010 \ldots 10101 \quad \text { or } \\
& \beta^{\prime}=0.0101 \ldots 10110101 \ldots 10101,
\end{aligned}
$$

or that

$$
\beta^{\prime} \rightarrow 0.0101 \ldots 011
$$

by the second or fourth transformation, i.e.

$$
\begin{aligned}
& \beta^{\prime}=0.1010 \ldots 010010 \ldots 1011 \quad \text { or } \\
& \beta^{\prime}=0.1010 \ldots 101101 \ldots 1011 .
\end{aligned}
$$

Further the double blocks $b_{i} b_{i+1}$ must be placed so that the "error term" in Lemma 3 becomes minimal. We carry this out for the two transformations yielding

$$
\beta^{\prime} \rightarrow 0.1010 \ldots 101
$$

(the second case is treated quite analogously).

If

$$
\beta^{\prime}=0.0101 \ldots 01001010 \ldots 10101
$$

then the "error term" has the form

$$
\frac{1}{3}\left(1-\frac{(-1)^{i}}{2^{i}}\right)(1-\tau)=: E(i)
$$


with

$$
\tau=0.1010 \ldots 101=\frac{2}{3}\left(1-\frac{1}{2^{s-i}}\right),
$$

and $i$ is odd. Hence

$$
E(i)=\frac{1}{9}\left(1+\frac{1}{2^{i}}\right)\left(1+\frac{1}{2^{s-1-i}}\right),
$$

which becomes minimal for $i=(s-1) / 2$, with value

$$
E=\frac{1}{9}\left(1+\frac{1}{2^{(s-1) / 2}}\right)^{2}
$$

If

$$
\beta^{\prime}=0.0101 \ldots 10110101 \ldots 10101
$$

then

$$
E(i)=\frac{1}{3}\left(1-\frac{(-1)^{i}}{2^{i}}\right) \tau
$$

with

$$
\tau=0.0101 \ldots 10101=\frac{1}{3}\left(1-\frac{1}{2^{s-i-1}}\right),
$$

and $i$ is even. Hence

$$
E(i)=\frac{1}{9}\left(1-\frac{1}{2^{i}}\right)\left(1-\frac{1}{2^{s-1-i}}\right),
$$

which becomes minimal for $i=2$ and for $i=s-3$ (note that $i=s-1$ would give one of the $\beta_{0}$ and $\left.E(i)=0\right)$, with value

$$
E=\frac{1}{12}\left(1-\frac{8}{2^{s}}\right)
$$

which is smaller than the $E$ above.

By also dealing with the second case we find that this is the minimal possible value for $E$ and we have found the first two values of $\beta^{\prime}$. The third value for $\beta^{\prime}$ is found by treating the second case.

The minimal error term $E$ also determines the value for

$$
\begin{aligned}
\sum_{u=0}^{s-1}\left\|2^{u} \beta^{\prime}\right\| & =\sum_{u=0}^{s-1}\left\|2^{u} \beta_{0}\right\|-E=\frac{s}{3}+\frac{1}{9}+\frac{1}{9 \cdot 2^{s}}-\frac{1}{12}\left(1-\frac{8}{2^{s}}\right) \\
& =\frac{s}{3}+\frac{1}{36}+\frac{7}{9 \cdot 2^{s}} .
\end{aligned}
$$

The case of $s$ even is dealt with quite analogously.

We again obtain a corollary: 
Corollary 2. The maximum

$$
\max _{\beta s-b i t}\left(\beta+\sum_{u=0}^{s-1}\left\|2^{u} \beta\right\|\right)=\frac{s}{3}+\frac{7}{9}+(-1)^{s} \frac{1}{9 \cdot 2^{s-1}}
$$

is attained if and only if $\beta$ is of the form

$$
\beta_{0}=\frac{2}{3}\left(1-\left(-\frac{1}{2}\right)^{s+1}\right) \quad \text { or } \quad \beta_{0}=\frac{5}{6}-\frac{1}{3}\left(-\frac{1}{2}\right)^{s} \text {. }
$$

REMARK 7. Note that here

$$
\beta_{0}=0.110101 \ldots 101 \quad \text { or } \quad \beta_{0}=0.101010 \ldots 011
$$

if $s$ is even and

$$
\beta_{0}=0.101010 \ldots 101 \quad \text { or } \quad \beta_{0}=0.110101 \ldots 011
$$

if $s$ is odd.

Proof of Corollary 2. If $\beta<1 / 2$ then we replace $\beta$ by $\beta+1 / 2$ and we obtain a larger value for the sum in question. So we can assume $\beta=$ $0.1 b_{2} b_{3} \ldots b_{s}$, and we note that $\beta+\|\beta\|=1$ always. So we have to maximize $\sum_{u=0}^{s-2}\left\|2^{u}(2 \beta)\right\|$. By Theorem 3(a) the result follows.

For later use (proof of Theorem 4(a)) we need a further type of "spectrum" result, namely Lemma 5. To prove it, we will use Lemma 4.

Lemma 4. Let $0 \leq \kappa<1$. Then

(a) The maximum

$$
\max _{\beta s-b i t}\left(\kappa \beta+\sum_{u=0}^{s-1}\left\|2^{u} \beta\right\|\right)=: \Sigma_{s}^{\kappa}
$$

is attained by

$$
\beta= \begin{cases}0.1010 \ldots 1011 & \text { for s even } \\ 0.1010 \ldots 101 & \text { for } s \text { odd }\end{cases}
$$

(b) The maximum

$$
\max _{\beta s-b i t}\left(-\kappa \beta+\sum_{u=0}^{s-1}\left\|2^{u} \beta\right\|\right)=: \Sigma_{s}^{-\kappa}
$$

is attained by

$$
\beta= \begin{cases}0.0101 \ldots 0101 & \text { for } s \text { even } \\ 0.0101 \ldots 011 & \text { for s odd }\end{cases}
$$

Proof. (a) We must have $b_{1}=1$, otherwise $1-\beta$ gives a larger value than $\beta$. We proceed by induction on $s$. For $s=1,2,3$ the assertion is easily 
checked. Now (since $\left.b_{1}=1\right)$

$$
\begin{aligned}
\Sigma_{s+2}^{\kappa} & =\max _{\beta s+2 \text {-bit }}\left(\kappa \beta+\sum_{u=0}^{s+1}\left\|2^{u} \beta\right\|\right) \\
& =\max _{\beta^{\prime} s+1 \text {-bit }}\left(\frac{\kappa+1}{2}+\beta^{\prime}\left(\frac{\kappa-1}{2}\right)+\sum_{u=0}^{s}\left\|2^{u} \beta^{\prime}\right\|\right) .
\end{aligned}
$$

Now $(\kappa-1) / 2<0$, so $b_{1}^{\prime}$ must be zero, otherwise $1-\beta^{\prime}$ would give a larger value. Hence $\beta^{\prime}=\beta^{\prime \prime} / 2$ with $\beta^{\prime \prime} s$-bit, and therefore

$$
\Sigma_{s+2}^{\kappa}=\frac{\kappa+1}{2}+\max _{\beta^{\prime \prime} s \text {-bit }}\left(\beta^{\prime \prime}\left(\frac{\kappa+1}{4}\right)+\sum_{u=0}^{s-1}\left\|2^{u} \beta^{\prime \prime}\right\|\right) .
$$

By the induction hypothesis the result follows.

(b) Set $\gamma=1-\beta$. Then

$$
-\gamma \kappa+\sum_{u=0}^{s-1}\left\|2^{u} \gamma\right\|=-\kappa+\kappa \beta+\sum_{u=0}^{s-1}\left\|2^{u} \beta\right\|
$$

and by part (a) the result follows.

The next lemma is of independent interest. Note for example that $1 / 4$ is the "average value" for $\|x\|$.

LEMmA 5. $\max _{\substack{\beta s-b i t \\ 0 \leq u_{0} \leq s-1}} \sum_{\substack{u=0 \\ u \neq u_{0}}}^{s-1}\left\|2^{u} \beta\right\|=\max _{\beta s-b i t} \sum_{u=0}^{s-1}\left\|2^{u} \beta\right\|-\frac{1}{4}$.

Proof. For $u_{0}$ fixed let

$$
\Sigma_{u_{0}}(\beta):=\sum_{\substack{u=0 \\ u \neq u_{0}}}^{s-1}\left\|2^{u} \beta\right\| \text { and } \Sigma_{u_{0}}\left(\beta_{0}\right):=\max _{\beta s \text {-bit }} \Sigma_{u_{0}}(\beta) .
$$

By Lemma $3, \beta_{0}$ must be of the form

$$
\beta_{0}=0.0101 \ldots b_{u_{0}+1} b_{u_{0}+2} \ldots b_{s} \quad \text { or } \quad \beta_{0}=0.1010 \ldots b_{u_{0}+1} b_{u_{0}+2} \ldots b_{s} .
$$

Let

$$
\bar{\beta}_{0}:=0 . b_{1} \ldots b_{u_{0}+1} \quad \text { and } \quad \widetilde{\beta}_{0}:=0 . b_{u_{0}+2} \ldots b_{s} .
$$

Then

$$
\begin{aligned}
\Sigma_{u_{0}}\left(\beta_{0}\right) & =\sum_{u=0}^{u_{0}-1}\left\|2^{u} \bar{\beta}_{0}\right\|+\kappa \widetilde{\beta}_{0}+\sum_{u=u_{0}+1}^{s-1}\left\|2^{u} \beta_{0}\right\| \\
& =\sum_{u=0}^{u_{0}-1}\left\|2^{u} \bar{\beta}_{0}\right\|+\kappa \widetilde{\beta}_{0}+\sum_{u=0}^{s-u_{0}-2}\left\|2^{u} \widetilde{\beta}_{0}\right\|
\end{aligned}
$$

with $\kappa=\sum_{i=1}^{u_{0}}(-1)^{b_{i}} / 2^{u_{0}+2-i}$. If $b_{u_{0}}=0$ then $\kappa>0$, if $b_{u_{0}}=1$ then $\kappa<0$. 
So by Lemma 3 (see also Theorem 3 ) the form of $\bar{\beta}_{0}$, and by Lemma 4 and by $b_{u_{0}}$ the form of $\widetilde{\beta}_{0}$ is determined (note that the form of $b_{u_{0}+1}$ must be different from $b_{u_{0}}$ and hence is 0 in any case).

We have

$$
\widetilde{\beta}_{0}=\frac{1}{3}\left(1-\frac{(-1)^{s-u_{0}-1}}{2^{s-u_{0}-1}}\right)
$$

and

$$
\kappa=-\frac{1}{6}\left(1-\frac{(-1)^{u_{0}}}{2^{u_{0}}}\right) \quad \text { or } \quad \kappa=-\frac{1}{3}\left(1+\frac{(-1)^{u_{0}}}{2^{u_{0}+1}}\right)
$$

according to which value for $\bar{\beta}_{0}$ is chosen from Theorem 3 .

Since we want to maximize

$$
\Sigma_{u_{0}}\left(\beta_{0}\right)=\sum_{u=0}^{u_{0}-1}\left\|2^{u} \bar{\beta}_{0}\right\|+\kappa \widetilde{\beta}_{0}+\sum_{u=0}^{s-u_{0}-2}\left\|2^{u} \widetilde{\beta}_{0}\right\|,
$$

only the larger first value for $\kappa$ is of relevance. Inserting it yields

$$
\begin{aligned}
\max _{\beta s \text {-bit }}\left(\sum_{u=0}^{s-1}\left\|2^{u} \beta\right\|-\Sigma_{u_{0}}\left(\beta_{0}\right)\right) & \\
= & \frac{1}{18}\left(5+\frac{(-1)^{u_{0}}}{2^{u_{0}}}+\frac{(-1)^{s-u_{0}-1}}{2^{s-u_{0}-1}}+\frac{(-1)^{s-1}}{2^{s-2}}\right),
\end{aligned}
$$

which attains its minimal value $1 / 4$ for $u_{0}=s-2$ if $s$ is odd, and for $u_{0}=1$ if $s$ is even.

\section{The discrepancy of the Hammersley net and an improved} upper bound for the discrepancy of digital $(0, s, 2)$-nets. In Theorem 1 for $\alpha, \beta s$-bit we have given an explicit formula for the discrepancy function

$$
\Delta(\alpha, \beta)=A_{2^{s}}([0, \alpha) \times[0, \beta))-2^{s} \alpha \beta
$$

of a digital $(0, s, 2)$-net in base 2 .

Take now arbitrary $\alpha^{\prime}, \beta^{\prime}$ with

$$
\alpha-\frac{1}{2^{s}}<\alpha^{\prime} \leq \alpha \quad \text { and } \quad \beta-\frac{1}{2^{s}}<\beta^{\prime} \leq \beta .
$$

Then (since all coordinates of the points of a digital net are $s$-bit) we have

$$
\Delta\left(\alpha^{\prime}, \beta^{\prime}\right)=\Delta(\alpha, \beta)-2^{s}\left(\alpha^{\prime} \beta^{\prime}-\alpha \beta\right),
$$

hence for the star-discrepancy $D_{N}^{*}$ of the net we have

$$
\left|D_{N}^{*}-\frac{1}{N} \max _{\alpha, \beta s \text {-bit }} \Delta(\alpha, \beta)\right|<\frac{2}{N}-\frac{1}{N^{2}}
$$

(note that $N=2^{s}$ ). 
We will call

$$
\frac{1}{N} \max _{\alpha, \beta s \text {-bit }} \Delta(\alpha, \beta)=: D_{N}^{\mathrm{d}}
$$

the discrete discrepancy of the net. $D_{N}^{\mathrm{d}}$ differs from $D_{N}^{*}$ at most by the almost negligible quantity $2 / N$ and seems for nets to be the more natural measure for the irregularities of distribution.

For a sequence of digital $(0, s, 2)$-nets, $s=1,2, \ldots, N=2^{s}$, we have

$$
\limsup _{N \rightarrow \infty} \frac{N D_{N}^{*}}{\log N}=\limsup _{N \rightarrow \infty} \frac{N D_{N}^{\mathrm{d}}}{\log N}
$$

(the same holds for liminf and for lim if it exists).

But if we want to obtain "exact results" the quantity $D_{N}^{\mathrm{d}}$ in spite of the minimal difference is much easier to handle than $D_{N}^{*}$.

This is clearly illustrated by the proof of the following theorem, in which we give the exact value of $D_{N}^{\mathrm{d}}$ and of $D_{N}^{*}$ for the Hammersley net and the exact places where they are attained. For $D_{N}^{\mathrm{d}}$ we moreover give the "second successive maxima" and the exact places where they are attained. The proof for $D_{N}^{\mathrm{d}}$ is much shorter than the one for $D_{N}^{*}$.

In [4] Halton and Zaremba claim that they give the exact value of $D_{N}^{*}$, but they only give a vague hint on how to prove the extremality of the extremal intervals. Entacher [3] uses their result.

Theorem 4. (a) For the discrete discrepancy $D_{N}^{\mathrm{d}}$ of the Hammersley net with $N=2^{s}$ points we have

$$
N D_{N}^{\mathrm{d}}=\max _{\alpha, \beta s-b i t} \Delta(\alpha, \beta)=\frac{s}{3}+\frac{1}{9}-\frac{(-1)^{s}}{9 \cdot 2^{s}}
$$

and the maximum will be attained if and only if $\alpha, \beta$ are of the form $\alpha_{0}, \beta_{0}$ with:

- for $s$ odd,

$$
\alpha_{0}=0.0101 \ldots 1011, \quad \beta_{0}=0.1010 \ldots 0101
$$

or

$$
\alpha_{0}=0.1010 \ldots 0101, \quad \beta_{0}=0.0110 \ldots 1011,
$$

- for s even,

$$
\alpha_{0}=\beta_{0}=0.1010 \ldots 1011 \quad \text { or } \quad \alpha_{0}=\beta_{0}=0.0101 \ldots 0101 \text {. }
$$

The second successive maximum for $\Delta(\alpha, \beta)(\alpha, \beta$ s-bit) is given by

$$
\max _{\substack{\alpha, \beta s-b i t \\(\alpha, \beta) \neq\left(\alpha_{0}, \beta_{0}\right)}} \Delta(\alpha, \beta)=\frac{s}{3}+\frac{1}{36}-(-1)^{s} \frac{7}{9 \cdot 2^{s}}
$$

and the places where this is attained can easily be obtained from the proof and from Theorem 3(b). 
(b) For the star-discrepancy $D_{N}^{*}$ of the Hammersley net with $N=2^{s}$ points we have

$$
N D_{N}^{*}=\frac{s}{3}+\frac{13}{9}-(-1)^{s} \frac{4}{9 \cdot 2^{s}}
$$

and the maximum is attained if and only if $\alpha, \beta$ are of the form $\alpha_{0}, \beta_{0}$ with:

- for $s$ odd,

$$
\alpha_{0}=0.1010 \ldots 10111, \quad \beta_{0}=0.1101 \ldots 01011
$$

or

$$
\alpha_{0}=0.1101 \ldots 01011, \quad \beta_{0}=0.1010 \ldots 10111,
$$

- for s even,

$$
\alpha_{0}=\beta_{0}=0.1010 \ldots 01011 \quad \text { or } \quad \alpha_{0}=\beta_{0}=0.1101 \ldots 10111
$$

for $s \geq 4$. For $s \leq 3$ the extremal values $\left(\alpha_{0}, \beta_{0}\right)$ are $(1 / 2,1 / 2)(s=1)$, $(3 / 4,3 / 4)(s=2)$ and $(7 / 8,7 / 8)(s=3)$.

Let us first draw a further consequence from the result and let us defer the proof of Theorem 4 to the end of this section.

As an almost immediate consequence we get the following bound for the discrepancy of digital $(0, s, 2)$-nets in base 2 , which improves the bounds (1) and (2).

TheOREM 5. For the star-discrepancy $D_{N}^{*}$ of a digital $(0, s, 2)$-net in base 2 we have

$$
N D_{N}^{*} \leq \frac{s}{3}+\frac{19}{9} \text {. }
$$

This bound is (by Theorem 4(b)) up to the summand 19/9 (which could be improved to $15 / 9)$ best possible.

In particular,

$$
\lim _{N \rightarrow \infty} \max \frac{N D_{N}^{*}}{\log N}=\frac{1}{3 \log 2}=0.4808 \ldots
$$

where the maximum is taken over all digital $(0, s, 2)$-nets in base 2 .

The value $1 /(3 \log 2)$ is attained for example for the sequence of Hammersley nets.

Proof. We have

$$
D_{N}^{*} \leq D_{N}^{\mathrm{d}}+\frac{2}{N}-\frac{1}{N^{2}}
$$

hence by Theorems 1 and 3 ,

$$
N D_{N}^{*} \leq 2+\max _{\beta s \text {-bit }} \sum_{u=0}^{s-1}\left\|2^{u} \beta\right\|-\frac{1}{2^{s}} \leq \frac{s}{3}+\frac{19}{9} .
$$


From this and from Theorem 4,

$$
\lim _{N \rightarrow \infty} \max \frac{N D_{N}^{*}}{\log N}=\frac{1}{3 \log 2}
$$

For the proof of part (b) of Theorem 4 we need some notation:

REMARK 8. For

$$
\alpha=0 . a_{1} \ldots a_{t} \ldots a_{s}, \quad \beta=0 . b_{1} \ldots b_{s-t} \ldots b_{s}
$$

we define

$$
\begin{array}{ll}
\alpha_{t}:=0 . a_{1} \ldots a_{t}, & \beta_{t}:=0 . b_{s+1-t} \ldots b_{s}, \\
\bar{\alpha}_{t}:=0 . a_{t+1} \ldots a_{s}, & \bar{\beta}_{t}:=0 . b_{1} \ldots b_{s-t} .
\end{array}
$$

Further, set

$$
\Sigma_{s}(\alpha, \beta):=\sum_{u=0}^{s-1}\left\|2^{u} \beta\right\| \sigma(u) \quad \text { with } \quad \sigma(u):=a_{s-u} \oplus a_{s+1-j(u)} .
$$

In $\sigma(u)$ we usually set $a_{s+1-j(u)}=0$ as long as $j(u)=0$. If in this case we alternatively set $a_{s+1-j(u)}:=1$ then we denote the corresponding sum by $\Sigma_{s}^{1}(\alpha, \beta)$.

Further we define

$$
T_{s}(\alpha, \beta):=\alpha+\beta+\Sigma_{s}(\alpha, \beta) .
$$

For $\kappa, \tau \in \mathbb{R}$ we more generally define

$$
T_{s}^{\tau, \kappa}(\alpha, \beta):=\tau \alpha+\kappa \beta+\Sigma_{s}(\alpha, \beta) .
$$

Now

$$
\begin{aligned}
T_{s}(\alpha, \beta) & =\alpha+\beta+\Sigma_{s}(\alpha, \beta) \\
& =\alpha+\beta+\Sigma_{s-t}\left(\bar{\alpha}_{t}, \bar{\beta}_{t}\right)+\beta_{t} \sum_{u=0}^{s-t-1} \frac{(-1)^{b_{u+1}}}{2^{s-t-u}} \sigma(u)+\widetilde{\Sigma}_{t}\left(\alpha_{t}, \beta_{t}\right) .
\end{aligned}
$$

Here $\widetilde{\Sigma}_{t}\left(\alpha_{t}, \beta_{t}\right)$ is either $\Sigma_{t}\left(\alpha_{t}, \beta_{t}\right)$ or $\Sigma_{t}^{1}\left(\alpha_{t}, \beta_{t}\right)$.

Since $\alpha=\alpha_{t}+\frac{1}{2^{t}} \bar{\alpha}_{t}$ and $\beta=\bar{\beta}_{t}+\frac{1}{2^{s-t}} \beta_{t}$ we get

$$
T_{s}(\alpha, \beta)=T_{s-t}^{\tau, 1}\left(\bar{\alpha}_{t}, \bar{\beta}_{t}\right)+\widetilde{T}_{t}^{1, \kappa_{t}}\left(\alpha_{t}, \beta_{t}\right),
$$

where $\widetilde{T}$ is defined via $\widetilde{\Sigma}$ instead of $\Sigma$, and $\tau=1 / 2^{t}$, and

$$
\kappa_{t}=\frac{1}{2^{s-t}}+\sum_{u=0}^{s-t-1} \frac{(-1)^{b_{u+1}}}{2^{s-t-u}} \sigma(u)
$$

Here it is important to note that $\kappa$ only depends on the form of $\bar{\alpha}_{t}$ and $\bar{\beta}_{t}$.

Let us consider for example $t=6$. Then it is an easy task to show with the help of MATHEMATiCA that for all $d \in\left\{0, \ldots, 2^{6}-1\right\}$ we have

$$
\left|\max _{\alpha_{6}, \beta_{6}} T_{6}^{1, d / 2^{6}}\left(\alpha_{6}, \beta_{6}\right)-\max _{\alpha_{6}, \beta_{6}} \widetilde{T}_{6}^{1, d / 2^{6}}\left(\alpha_{6}, \beta_{6}\right)\right| \leq 1 / 2^{6} .
$$


Hence for all $\kappa$

$$
\left|\max _{\alpha_{6}, \beta_{6}} T_{6}^{1, \kappa}\left(\alpha_{6}, \beta_{6}\right)-\max _{\alpha_{6}, \beta_{6}} \widetilde{T}_{6}^{1, \kappa}\left(\alpha_{6}, \beta_{6}\right)\right|<1 / 2^{5} .
$$

Further we need the following lemma:

Lemma 6. If

$$
T_{s}\left(\alpha_{0}, \beta_{0}\right)=\max _{\alpha, \beta s-b i t} T_{s}(\alpha, \beta),
$$

then $\beta_{0}$ has at most three consecutive equal digits $b_{i} b_{i+1} b_{i+2}, i \geq 2$, in its base 2 representation.

Proof. First we note that the first digit of $\beta_{0}$ must be one, otherwise replacing $\beta_{0}$ by $\beta_{0}+1 / 2$ and choosing a suitable $\alpha_{0}$ gives a larger value $T$.

Then we note that, as is easily calculated, the special choice

$$
\alpha^{\prime}=0.101 \ldots 1011, \quad \beta^{\prime}=0.101 \ldots 1011
$$

if $s$ is even and

$$
\alpha^{\prime}=0.1101 \ldots 1011, \quad \beta^{\prime}=0.1010 \ldots 0111
$$

if $s$ is odd gives the value

$$
T_{s}\left(\alpha^{\prime}, \beta^{\prime}\right)=\frac{s}{3}+\frac{13}{9}+\frac{1}{2^{s}}-(-1)^{s} \frac{4}{9} \cdot \frac{1}{2^{s}} .
$$

Assume now on the contrary that $\beta_{0}$ has at least four equal digits $b_{i} b_{i+1} b_{i+2} b_{i+3}, i \geq 2$, in its base 2 representation. Assume these are ones (the other case is handled in the same way). Then

$$
T_{s}\left(\alpha_{0}, \beta_{0}\right) \leq 1+\beta_{0}+\sum_{u=0}^{s-1}\left\|2^{u} \beta_{0}\right\| .
$$

Now we can apply some of the transformations from Corollary 1 to $\beta_{0}$ until $b_{i} b_{i+1}$ is the first block of equal digits (with $i \geq 2$ ). Therefore

$$
\beta_{0}+\sum_{u=0}^{s-1}\left\|2^{u} \beta_{0}\right\|
$$

will not decrease. Now we can apply two times one of the last two transformations from Corollary 1 to $b_{i} b_{i+1}$ and then to $b_{i+1} b_{i+2}$. Note that $\tau \geq 3 / 4$ in the first application and $\tau \geq 1 / 2$ in the second. Therefore

increases at least by

$$
\beta_{0}+\sum_{u=0}^{s-1}\left\|2^{u} \beta_{0}\right\|
$$

$$
\frac{1}{3} \cdot \frac{3}{4}\left(1-\frac{(-1)^{i-1}}{2^{i-1}}\right)+\frac{1}{3} \cdot \frac{1}{2}\left(1-\frac{(-1)^{i}}{2^{i}}\right)=\frac{5}{12}+\frac{(-1)^{i}}{3 \cdot 2^{i}} \geq \frac{3}{8} .
$$

Hence we have, by the remark at the beginning of this proof and by Corollary 2 , 


$$
\begin{aligned}
\frac{s}{3}+\frac{13}{9}+\frac{1}{2^{s}}-(-1)^{s} \frac{4}{9} \cdot \frac{1}{2^{s}} & \leq T_{s}\left(\alpha_{0}, \beta_{0}\right) \\
& \leq 1+\max _{\beta s \text {-bit }}\left(\beta+\sum_{u=0}^{s-1}\left\|2^{u} \beta\right\|\right)-\frac{3}{8} \\
& =\frac{5}{8}+\frac{s}{3}+\frac{7}{9}+(-1)^{s} \frac{2}{9 \cdot 2^{s}}
\end{aligned}
$$

hence

$$
\frac{1}{24}+\frac{1}{2^{s}}\left(1-\frac{2}{3}(-1)^{s}\right) \leq 0
$$

a contradiction.

REMARK 9. It is easy to show with the help of a $\mathrm{C}^{++}$program that the assertion of Theorem 4(b) holds for $s \leq 11$.

In fact it is not difficult to prove (with the help of Lemmas 5 and 6) that the extremal values $\alpha_{0}, \beta_{0}$ from Theorem 4(b) must have the property that $a_{s-u} \oplus a_{s+1-j(u)}=1$ for all $u=0, \ldots, s-1$. Hence for every $\beta_{0}$ there is only one possible $\alpha_{0}$. So it was easily possible to carry out the numerical calculation with MATHEMATICA.

Proof of Theorem 4. (a) We use Example 2. For a given $\beta$ the value

$$
\Delta(\alpha, \beta)=\sum_{u=0}^{s-1}\left\|2^{u} \beta\right\|\left(a_{s-u} \oplus a_{s+1-j(u)}\right)
$$

always becomes maximal if $\alpha$ is chosen such that $a_{s-u} \oplus a_{s+1-j(u)}=1$ for all $u$. Hence $D_{N}^{\mathrm{d}}$ is attained for the $\beta$ maximizing

$$
\sum_{u=0}^{s-1}\left\|2^{u} \beta\right\|
$$

(those are provided by Theorem 3) and the corresponding $\alpha$. This gives the values claimed in the result.

For the second successive maximum there are principally two possible cases: either $a_{s-u} \oplus a_{s+1-j(u)}=1$ for all $u$, and then $\beta$ must be of the form from Theorem 3(b), or $a_{s-u} \oplus a_{s+1-j(u)}=0$ for some $u$. But comparing Theorem 3 and Lemma 5 shows that only the first case can give the second successive maximum.

(b) For $\alpha, \beta s$-bit $\Delta(\alpha, \beta)$ always is positive by Example 2 . Hence $D_{N}^{*}$ will certainly be attained for intervals of the form

$$
\left[0, \alpha-1 / 2^{s}\right] \times\left[0, \beta-1 / 2^{s}\right]
$$

with $\alpha, \beta s$-bit, and therefore

$$
N D_{N}^{*}=\max _{\alpha, \beta s \text {-bit }}(\Delta(\alpha, \beta)+\alpha+\beta)-1 / 2^{s}
$$


(see Remark 2). By Remark 9 it suffices to assume that $s \geq 12$. Let $\alpha^{(0)}, \beta^{(0)}$ be such that

$$
T_{s}\left(\alpha^{(0)}, \beta^{(0)}\right)=\max _{\alpha, \beta s \text {-bit }} T_{s}(\alpha, \beta)
$$

By Lemma $6, \beta^{(0)}$ has at most three consecutive equal digits (after the first place) and the first digit $b_{1}$ of $\beta^{(0)}$ is 1 . Assume there is a $u \leq s-12$ with $\sigma(u)=0$ (see Remark 8 for the notations here and in the following), and let $u_{0}$ be maximal with this property. Then change $a_{s-u_{0}}, \ldots, a_{7}$ so that $\sigma\left(u_{0}\right)$ becomes 1 and $\sigma\left(u_{0}+1\right), \ldots, \sigma(s-7)$ remain unchanged. Thereby $\kappa_{6}$ changes at most by $1 / 2^{s-6-u_{0}} \leq 1 / 2^{6}$. Finally choose $a_{6}, \ldots, a_{1}$ and $b_{s-5}, \ldots, b_{s}$ so that $\widetilde{T}^{1, \kappa_{6}}\left(\alpha_{6}^{\prime}, \beta_{6}^{\prime}\right)$ becomes maximal for the new values $\alpha^{\prime}, \beta^{\prime}$. Then (see Remark 8),

$$
T_{s}\left(\alpha^{\prime}, \beta^{\prime}\right)=T_{s-6}^{\tau, 1}\left(\bar{\alpha}_{6}^{\prime}, \bar{\beta}_{6}^{\prime}\right)+\widetilde{T}_{6}^{1, \kappa_{6}^{\prime}}\left(\alpha_{6}^{\prime}, \beta_{6}^{\prime}\right)
$$

(note that we obtain a new summand of value at least $1 / 4$, but $\alpha$ may decrease to almost zero)

$$
\geq T_{s-6}^{\tau, 1}\left(\bar{\alpha}_{6}^{(0)}, \bar{\beta}_{6}^{(0)}\right)+\frac{1}{4}-\tau-\left|\kappa_{6}^{\prime}-\kappa_{6}\right|+\widetilde{T}_{6}^{1, \kappa_{6}}\left(\alpha_{6}^{\prime}, \beta_{6}^{\prime}\right)
$$

(by the numerical result in Remark 8; note that the tilde on $\widetilde{T}$ is here related to $\alpha^{\prime}, \beta^{\prime}$ and in the following line to $\left.\alpha^{(0)}, \beta^{(0)}\right)$

$$
\begin{aligned}
& \geq T_{s-6}^{\tau, 1}\left(\bar{\alpha}_{6}^{(0)}, \bar{\beta}_{6}^{(0)}\right)+\frac{1}{4}-\tau-\frac{1}{2^{6}}+\widetilde{T}_{6}^{1, \kappa_{6}^{\prime}}\left(\alpha_{6}^{(0)}, \beta_{6}^{(0)}\right)-\frac{1}{2^{5}} \\
& >T_{s}\left(\alpha^{(0)}, \beta^{(0)}\right)+\frac{1}{2^{4}}-\frac{4}{2^{6}} \\
& =T_{s}\left(\alpha^{(0)}, \beta^{(0)}\right)
\end{aligned}
$$

a contradiction. Hence

$$
\begin{aligned}
T_{s}\left(\alpha^{(0)}, \beta^{(0)}\right)= & \beta^{(0)}+\sum_{u=0}^{s-12}\left\|2^{u} \beta^{(0)}\right\| \\
& +\frac{1}{2^{11}} \bar{\alpha}_{11}^{(0)}+\alpha_{11}^{(0)}+\widetilde{\Sigma}_{11}\left(\alpha_{11}^{(0)}, \beta_{11}^{(0)}\right) .
\end{aligned}
$$

Therefore by Corollary $1, b_{1}^{(0)}, \ldots, b_{s-11}^{(0)}$ and $a_{12}^{(0)}, \ldots, a_{s}^{(0)}$ must be of the form (we concentrate on " $s$ odd", " $s$ even" being carried out quite analogously)

$$
\bar{\beta}_{11}^{(0)}=0.110101 \ldots 01, \quad \bar{\alpha}_{11}^{(0)}=0.0101 \ldots 0111
$$

or

$$
\bar{\beta}_{11}^{(0)}=0.1010 \ldots 011, \quad \bar{\alpha}_{11}^{(0)}=0.0101 \ldots 011 .
$$

So it remains to maximize $\widetilde{T}_{11}^{1, \kappa}\left(\alpha_{11}, \beta_{11}\right)$. 
In the first case we have

$$
\left|\kappa+\frac{1}{3}\left(1-\frac{1}{2^{12}}\right)\right|<\frac{1}{2^{13}},
$$

in the second case we have

$$
\left|\kappa-\frac{1}{3}\left(1-\frac{1}{2^{12}}\right)\right|<\frac{1}{2^{13}},
$$

so it suffices to maximize

$$
\widetilde{T}_{11}^{1,-\frac{1}{3}\left(1-1 / 2^{12}\right)}\left(\alpha_{11}, \beta_{11}\right) \quad \text { respectively } \widetilde{T}_{11}^{1, \frac{1}{3}\left(1-1 / 2^{12}\right)}\left(\alpha_{11}, \beta_{11}\right) .
$$

This is easily done with a MAтнематісA program and the result follows.

5. A class of nets with smaller star-discrepancy. We have seen in Theorem 5 that the Hammersley net essentially is the "worst" distributed digital $(0, s, 2)$-net in base 2 .

We will show here that the star-discrepancy of the nets generated by

$$
C_{1}=\left(\begin{array}{ccccc}
1 & 0 & \ldots & 0 & 0 \\
0 & 1 & \ldots & 0 & 0 \\
\ldots & \ldots & \ldots & \ldots & \ldots \\
0 & 0 & \ldots & 1 & 0 \\
0 & 0 & \ldots & 0 & 1
\end{array}\right) \quad \text { and } C_{2}=\left(\begin{array}{ccccc}
1 & 1 & \ldots & 1 & 1 \\
1 & 1 & \ldots & 1 & 0 \\
\ldots & \ldots & \ldots & \ldots & \ldots \\
1 & 1 & \ldots & 0 & 0 \\
1 & 0 & \ldots & 0 & 0
\end{array}\right)
$$

is essentially smaller. Indeed it seems, by numerical experiments carried out by Entacher, that these nets are the essentially best distributed digital $(0, s, 2)$-nets in base 2 . We have

TheOREM 6. For the star-discrepancy $D_{N}^{*}$ of the digital net in base 2 generated by

$$
C_{1}=\left(\begin{array}{ccccc}
1 & 0 & \ldots & 0 & 0 \\
0 & 1 & \ldots & 0 & 0 \\
\ldots & \ldots & \ldots & \ldots & \ldots \\
0 & 0 & \ldots & 1 & 0 \\
0 & 0 & \ldots & 0 & 1
\end{array}\right) \quad \text { and } \quad C_{2}=\left(\begin{array}{ccccc}
1 & 1 & \ldots & 1 & 1 \\
1 & 1 & \ldots & 1 & 0 \\
\ldots & \ldots & \ldots & \ldots & \ldots \\
1 & 1 & \ldots & 0 & 0 \\
1 & 0 & \ldots & 0 & 0
\end{array}\right)
$$

we have

$$
\frac{N D_{N}^{*}}{s} \geq 0.2
$$

for all $N\left(N=2^{s}\right)$ and

$$
\limsup _{N \rightarrow \infty} \frac{N D_{N}^{*}}{s} \leq 0.226341 \ldots
$$

REMARK 10. Hence for these nets we have

$$
0.2885 \ldots=\frac{1}{5 \log 2} \leq \liminf _{N \rightarrow \infty} \frac{N D_{N}^{*}}{\log N} \leq \limsup _{N \rightarrow \infty} \frac{N D_{N}^{*}}{\log N} \leq 0.32654 \ldots
$$


Indeed we conjecture that

$$
\lim _{N \rightarrow \infty} \frac{N D_{N}^{*}}{\log N}=\frac{1}{5 \log 2},
$$

and that this is the best possible value at all, i.e.

$$
\lim _{N \rightarrow \infty} \min \frac{N D_{N}^{*}}{\log N}=\frac{1}{5 \log 2}
$$

where the minimum is taken over all digital $(0, s, 2)$-nets in base 2 .

Proof of Theorem 6. We will show that the lower bound even holds for

$$
\max _{\alpha, \beta s \text {-bit }} \Delta(\alpha, \beta)
$$

and also for the upper bound it suffices to consider $\Delta(\alpha, \beta)$ for $\alpha, \beta$ s-bit. Recall from Example 3 that for $\alpha, \beta s$-bit we have

$$
\begin{aligned}
\Delta(\alpha, \beta) & =\sum_{u=0}^{s-1}\left\|2^{u} \beta\right\|(-1)^{a_{1}+\ldots+a_{s-u}} \frac{(-1)^{a_{s-u}}-(-1)^{a_{s+1-j(u)}}}{2} \\
& =\sum_{u=0}^{s-1}\left\|2^{u} \beta\right\|(-1)^{a_{1}+\ldots+a_{s-u-1}}\left(a_{s-u} \oplus a_{s+1-j(u)}\right),
\end{aligned}
$$

where

$$
j(u):=\left\{\begin{array}{l}
0 \quad \text { if } u=0, \\
0 \quad \text { if } a_{1} \oplus \ldots \oplus a_{s+1-j}=b_{j} \text { for } j=1, \ldots, u, \\
\max \left\{j \leq u: a_{1} \oplus \ldots \oplus a_{s+1-j} \neq b_{j}\right\} \text { otherwise. }
\end{array}\right.
$$

We set $\widetilde{a}_{i}:=a_{1} \oplus \ldots \oplus a_{s+1-i}$ and $\widetilde{\alpha}:=0 . \widetilde{a}_{1} \ldots \widetilde{a}_{s}$. Then

$$
a_{s+1-i}=\widetilde{a}_{i} \oplus \tilde{a}_{i+1}, \quad a_{s+1-j(u)}=\widetilde{a}_{r(u)} \oplus \widetilde{a}_{r(u)+1},
$$

where

$$
r(u):= \begin{cases}0 & \text { if } u=0, \\ 0 & \text { if } b_{j}=\widetilde{a}_{j} \text { for } j=1, \ldots, u, \\ \max \left\{r \leq u: b_{j} \neq \widetilde{a}_{j}\right\} & \text { otherwise }\end{cases}
$$

and where we have to set $\widetilde{a}_{r(u)} \oplus \widetilde{a}_{r(u)+1}:=0$ if $r(u)=0$ and $\widetilde{a}_{s+1}:=0$. Then

$$
\Delta(\alpha, \beta)=\sum_{u=0}^{s-1}\left\|2^{u} \beta\right\| \varrho(u)=: \delta(\widetilde{\alpha}, \beta),
$$

where

$$
\varrho(u):=(-1)^{\widetilde{a}_{u+2}}\left(\widetilde{a}_{u+1} \oplus \widetilde{a}_{u+2} \oplus \widetilde{a}_{r(u)} \oplus \widetilde{a}_{r(u)+1}\right) .
$$

To obtain the lower bound consider

$$
\beta=0.00100010001 \ldots b_{s}, \quad \widetilde{\alpha}=0.10001000100 \ldots a_{s}
$$


with the exception that $b_{s}=1$ instead of 0 if $s=4 l+1$ or $s=4 l+2$. Then

$$
\varrho(u)= \begin{cases}-1 & \text { if } u=4 l+3 \\ 1 & \text { otherwise }\end{cases}
$$

with the only exception that $\varrho(s-1)=0$ if $s=4 l$. Then

$$
\beta=\sum_{i=0}^{[s / 4]-1} \frac{1}{2^{4 i+3}}+\frac{b_{s}}{2^{s}}
$$

hence

$$
\left\|2^{u} \beta\right\|=\sum_{i=\lceil u / 4-1 / 2\rceil}^{[s / 4]-1} \frac{1}{2^{4 i+3-u}}+\frac{b_{s}}{2^{s-u}}
$$

for $u \neq 4 l+2$ and it is 1 minus this quantity if $u=4 l+2$. So

$$
\delta(\widetilde{\alpha}, \beta)=\sum_{l=0}^{[(s-5) / 4]}\left(\left\|2^{4 l} \beta\right\|+\left\|2^{4 l+1} \beta\right\|+\left\|2^{4 l+2} \beta\right\|-\left\|2^{4 l+3} \beta\right\|\right)+R,
$$

with

$$
R= \begin{cases}1 / 2 & \text { if } s=4 l+1 \\ 3 / 4 & \text { if } s=4 l+2 \\ 7 / 8 & \text { else }\end{cases}
$$

Inserting for $\left\|2^{u} \beta\right\|$ and evaluating the resulting finite geometric series then yields

$$
\delta(\widetilde{\alpha}, \beta)=\frac{4}{5}\left[\frac{s-1}{4}\right]+\frac{16^{[(s-1) / 4]}-1}{16^{[s / 4]}} \cdot \begin{cases}2 / 25+7 / 8 & \text { if } s=4 l \\ (-11 / 50)+1 / 2 & \text { if } s=4 l+1 \\ (-7 / 100)+3 / 4 & \text { if } s=4 l+2 \\ 1 / 200+7 / 8 & \text { if } s=4 l+3 .\end{cases}
$$

Now it is a simple task to check that in each of the four cases $\delta(\widetilde{\alpha}, \beta) / s$ is decreasing to $1 / 5$, and so the lower bound follows.

To obtain the upper bound consider for given $r \in \mathbb{N}$ the quantity

$$
\delta_{r}:=\sup _{\alpha, \beta} \sum_{u=0}^{r-1} \varrho(u)\left\|2^{u} \beta\right\|,
$$

where the supremum is taken over all $\beta \in[0,1)$ and over all $r+1$-bit $\alpha=0 . a_{1} \ldots a_{r+1}$. (Note that this means that $a_{r+1}$ is not automatically set to 0 as is done for $r$-bit $\alpha$.)

This supremum is obviously attained (respectively approached) in the following form: let $u_{0}$ be the largest index such that $\varrho\left(u_{0}\right) \neq 0$; then $\varrho\left(u_{0}\right)=1$. Further the supremum is attained for some $\beta$ with $b_{r+1}=b_{r+2}=\ldots=0$ if $b_{u_{0}+1}=1$ and it is approached by $\beta$ with $b_{r+1}=b_{r+2}=\ldots=1$ if $b_{u_{0}+1}=0$. 
So it can be shown for example with Mathematica that

$$
\delta_{11}=\frac{5099}{2048}=2.48975 \ldots,
$$

and this value is attained with $b_{u_{0}+1}=1$.

Now for $s$ with $s=11 q+w, 0 \leq w \leq 10$, for all $\widetilde{\alpha}, \beta$ we have $\delta(\widetilde{\alpha}, \beta) \leq$ $q \delta_{11}+w$, hence

$$
\frac{\delta(\widetilde{\alpha}, \beta)}{s} \leq \frac{1}{s}\left[\frac{s}{11}\right] \cdot 2.48975 \ldots+\frac{10}{s}
$$

which tends to $0.226341 \ldots$ as $s \rightarrow \infty$, and the result follows.

\section{References}

[1] R. Béjian, Minoration de la discrépance d'une suite quelconque sur T, Acta Arith. 41 (1982), 185-202.

[2] L. de Clerck, A method for exact calculation of the stardiscrepancy of plane sets applied to the sequences of Hammersley, Monatsh. Math. 101 (1986), 261-278.

[3] K. Entacher, Haar function based estimates of the star-discrepancy of plane digital nets, ibid. 130 (2000), 99-108.

[4] J. H. Halton and S. K. Zaremba, The extreme and the $L^{2}$ discrepancies of some plane sets, ibid. 73 (1969), 316-328.

[5] G. Larcher, H. Niederreiter and W. Ch. Schmid, Digital nets and sequences constructed over finite rings and their application to quasi-Monte Carlo integration, ibid. 121 (1996), 231-253.

[6] G. Larcher and F. Pillichshammer, Walsh series analysis of the $L_{2}$-discrepancy of symmetrized point sets, ibid. 132 (2001), 1-18.

[7] G. Larcher and G. Pirsic, Base change problems for generalized Walsh series and multivariate numerical integration, Pacific J. Math. 189 (1999), 75-105.

[8] H. Niederreiter, Point sets and sequences with small discrepancy, Monatsh. Math. 104 (1987), 273-337.

Institut für Analysis

Universität Linz

Altenbergerstraße 69

A-4040 Linz, Austria

E-mail: gerhard.larcher@jku.at

friedrich.pillichshammer@jku.at

Received on 7.5.2001

and in revised form on 20.3.2002 